gr-qc/0207076

\title{
Group of boost and rotation transformations with two observer-independent scales
}

\author{
Nicola Rossano BRUNO \\ Dipartimento di Fisica, Università di Roma ROMA TRE, \\ Via Vasca Navale 84, 00146 Roma, Italy \\ I.N.F.N. - Sezione di ROMA TRE, Roma, Italy
}

\begin{abstract}
I examine the structure of the deformed Lorentz transformations in one of the recently-proposed schemes with two observer-independent scales. I develop a technique for the analysis of general combinations of rotations and deformed boosts. In particular, I verify explicitly that the transformations form group.
\end{abstract}

\section{INTRODUCTION}

Recently many modern approaches to the problem of unification of Quantum Mechanics and General Relativity have led to arguments that suggests a modification of Lorentz symmetry at the Planck Scale. As a possible tool for this ongoing quantum-gravity debate AmelinoCamelia proposed [1, 2] the possibility that the rotation/boost transformations between inertial observers might be characterized by two rather than one observerindependent scale: in addition to the velocity scale, $c$, one introduces a length (momentum) scale $\lambda(1 / \lambda)$. In Refs. 11, 2] an illustrative example of the new type of transformation laws was also analyzed, obtaining results, including the emergence of a maximum momentum $1 / \lambda$, in leading order in the second observer-independent scale $\lambda$. In Ref. 34 the analysis of that model was generalized to all orders in $\lambda$, and in particular it was verified that indeed the new transformation rules saturate at maximum momentum $1 / \lambda$.

In these past few months interest in this idea has increased as various authors [4, 5, 6, 7, 8] considered different ways to introduce the two observer-independent scales, still following the general scheme proposed in Refs. [1, 2]. These relativistic theories are being called "Doubly Special Relativity" (DSR), and their possible relevance for the study of noncommutative spacetimes and loop quantum gravity is under investigation 1, 国, 6. In the physics of quantum spacetime and quantum gravity DSR may provide a tool for the description of the Planck length as a kinematical scale of the structure of spacetime or energy-momentum space, upon identification of $\lambda$ with the Planck length: $\lambda \sim L_{p}$. The observable implications of DSR theories are being studied mostly in relation with forthcoming powerful Lorentz-symmetry tests [9, 10] and in searches of a kinematical solution for the puzzling observations of ultra-high-energy cosmic rays [11, 12, 13].

Also under investigation [1, 6, 7] is the role that $\kappa$ Poincaré Hopf algebras [14, 15, 16] can play in these DSR theories. In the one-particle sector of the DSR theories so far considered $\kappa$-Poincaré mathematics has a role which is analogous to the role of Lorentz/Poincaré mathematics in Einstein's Special Relativity. For the two-particle sector it is still unclear if and in which way $\kappa$-Poincaré could have a role.

The connection with $\kappa$-Poincaré may be cause of concern with respect to the group properties of the DSR transformations; in fact, for the Lorentz sector of $\kappa$ Poincaré Hopf algebras it is generally expected [17] that the transformations obtained by exponentiation of the boost/rotation generators would not form group: they would only form quasigroup in the sense of Batalin [18], As experimentalists are preparing [10] for investigations that have the sensitivity to test Planck-scale modifications of Lorentz symmetry, it is important to settle these possible concerns about the group structure, since they may affect the willingness of experimentalists to include DSR predictions among the tests they actually perform. If DSR symmetries only lead to quasi-group structure, as generally expected for theories based on $\kappa$-Poincaré mathematics, one might conclude that these symmetries are not suitable for the description of Planck-scale physics. In fact the structure coefficients of a quasi-group depend on the initial variables on which the transformations act. To characterize the puzzling implications of this dependence one can consider three inertial observers that are performing measures on the four-momentum of a particle. Quasi-group structure implies [18] that there must exist a unique transformation that relates the measures performed by the first and the third inertial observer, but this transformation depends on the momenta observed by the second inertial observer.

With respect to this issue I here reconsider the theory "DSR1", the one used as illustrative example in Refs. 11. 2]. In Ref. [1 it was argued that the specific $\kappa$-Poincaré Hopf algebra that is relevant for DSR1 is a special case in which the exponentiation of the generators in the Lorentz sector does lead to an ordinary symmetry group. The observation in Ref. [1] was based on the fact that the relevant $\kappa$-Poincaré Hopf algebra has a classical (undeformed) Lorentz sector: the commutation relations for rotation/boost generators are just the 
usual Lorentz ones, although the differential representation of the boost generators on energy-momentum is deformed (nonlinear). This in particular implies that the Batalin conditions 18] for group structure ${ }^{1}$ are satisfied. Since the algebra is the same, using the Baker-CampbellHausdorff formula one deduces [1] that by exponentiation of the DSR1 rotation/boost generators one obtains genuine group elements, which actually combine just as usual: one obtains again the Lorentz group, only realized nonlinearly.

Following the same line of analysis one can show that the other versions of $\kappa$-Poincaré Hopf algebras considered in the past form only quasi-group with the mentioned problem of physical interpretation.

However this argument based on $\mathrm{BCH}$ is not enough to establish that the path from the algebra to the group properties can be legitimately followed. There are some mathematical issues to be investigated, including the assumption that the $\mathrm{BCH}$ series converges (see e. $g$. Ref. 19 and the references therein). Rather than give these proofs I here settle the issue through an explicit verification of the group properties of the DSR1 transformations. In addressing this issue I also develop a technique for the analysis of DSR1 deformed boost/rotation transformations which should prove useful in applications of the formalism; in fact, the nonlinear structure of the DSR1 transformation rules of course prevents us from describing boosts through matrices and the calculations can in some cases become rather involved. The technique I introduce is useful in handling these difficulties.

In section II I revise some issues relevant for the BakerCampbell-Hausdorff (BCH) formula that emerge naturally in the composition of two exponential of operators that do not commute. Notice that in ordinary Special Relativity we do not need to resort to $\mathrm{BCH}$ because the Lorentz generators have a simple matrix representation. The group laws in that case simply are the matrix products while in the DSR1-deformed case we do not have a matrix representation and we have to directly deal with $\mathrm{BCH}$.

In section III I introduce a system of differential equations whose solutions are the deformed Lorentz transformations. To derive them I exploit a formalism 20] introduced by Feynman in 1951 as a tool for the analysis of QED perturbation theory.

In section IV I consider some explicit applications of the formulas obtained in Section III, with emphasis on the emergence of the expected group properties. Section $\mathrm{V}$ presents some closing remarks.

\footnotetext{
${ }^{1}$ Batalin basically observed [18] that an ordinary group will be obtained by exponentiation of the generators if the algebra truly closes on its generators. In generic $\kappa$-Poincaré Hopf algebras some commutators of the generators of the Lorentz sector are expressed in terms of translation generators, and this causes the transformations obtained by exponentiation of the Lorentz-sector generators to form only quasigroup rather than group [17, 18.
}

\section{GROUP PROPERTIES AND BAKER-CAMPBELL-HAUSDORFF FORMULA}

The boost and rotation generators adopted in the DSR1 scheme are [1, 3]:

$$
\begin{aligned}
& N_{i}=i k_{i} \frac{\partial}{\partial \omega}+i\left(\frac{\lambda}{2} \vec{k}^{2}+\frac{1-e^{-2 \lambda \omega}}{2 \lambda}\right) \frac{\partial}{\partial k_{i}}-i \lambda k_{i}\left(k_{j} \frac{\partial}{\partial k_{j}}\right) ; \\
& M_{i}=-i \epsilon_{i j k} k_{j} \frac{\partial}{\partial k_{k}}
\end{aligned}
$$

These generators can be naturally described as part of the so-called bicross-product-basis $\kappa$-Poincaré Hopf algebra 15, 16, which in the algebra sector ${ }^{2}$ prescribes the commutation rules

$$
\begin{aligned}
{\left[M_{\mu \nu}, M_{\rho \tau}\right] } & =i\left(\eta_{\mu \tau} M_{\nu \rho}-\eta_{\nu \rho} M_{\nu \tau}+\eta_{\nu \rho} M_{\mu \tau}-\eta_{\nu \tau} M_{\mu \rho}\right) \\
{\left[M_{i}, k_{j}\right] } & =i \epsilon_{i j k} k_{k} ; \quad\left[M_{i}, \omega\right]=0 ; \\
{\left[N_{i}, k_{j}\right] } & =i \delta_{i j}\left(\frac{1}{2 \lambda}\left(1-e^{-2 \lambda \omega}\right)+\frac{\lambda}{2} \vec{k}^{2}\right)-i \lambda k_{i} k_{j} \\
{\left[N_{i}, \omega\right] } & =i k_{i} \\
{\left[P_{\mu}, P_{\nu}\right] } & =0
\end{aligned}
$$

where $P_{\mu}=(\omega, \vec{k})$ are the translation generators and $M_{\mu \nu}$ are the Lorentz-sector generators with rotations given by $M_{k}=\frac{1}{2} \epsilon_{i j k} M_{i j}$ and boosts $N_{i}=M_{0 i}$.

The special feature of the bicross-product-basis is the fact that the Lorentz-sector commutators are undeformed, only the commutators between boosts and translation generators are deformed. This fact has important implications for the group properties of finite transformations.

The DSR1 finite rotation/boost transformations on energy-momentum space are constructed [i] by the action of the exponentiation of the generators. This action was first studied [1] 2] only in leading order in $\lambda$, and then, in Ref. [3], the exact (all orders in $\lambda$ ) form of the transformations was analyzed for the special case of the action on energy-momentum space of the exponentiation of one boost generator. Here I consider general rotation/boost transformations of the form

$$
P_{\mu}=e^{O} P_{\mu}^{0} e^{-O}
$$

where $O=-i \sum_{i} \xi_{i} O_{i}$ and $O_{i}=\left\{N_{1}, N_{2}, N_{3}, M_{1}, M_{2}, M_{3}\right\}$.

For the composition of two such transformations one can naturally [1], 2] resort to the Baker-CampbellHausdorff's formula (BCH)

$$
\begin{aligned}
H(A, B) & =A+B+\frac{1}{2}[A, B]+\frac{1}{12}[A,[A, B]]+ \\
& +\frac{1}{12}[B,[B, A]]-\frac{1}{24}[A,[B,[A, B]]]+\ldots
\end{aligned}
$$

\footnotetext{
2 The coalgebra sector, which plays no explicit role in my analysis, is described in Refs. 115, 16.
} 
where $A$ and $B$ are two operators with known commutation relations.

It is useful to consider a generic element of a Lie algebra $g \in \mathrm{G}$ such that $g=\sum_{i} \alpha_{i} g_{i}$ where $\left\{g_{i}\right\}$ are the generators of $\mathrm{G}$ and $\alpha_{i}$ complex numbers. One finds 19 that $\mathrm{G}$ is a group with product $H(\cdot, \cdot)$. In fact $\mathrm{BCH}$ introduces only commutators between algebra generators that are closed in the algebra by definition (2), and then $H(\cdot, \cdot)$ is a linear combination of generators with coefficients that only depend on the $\alpha_{i}$ and on the structure coefficients. Exploiting this fact one can easily prove that the transformations done by the exponentiation of a Lie algebra do form group with product done by multiplication of exponentials. The identity obviously is $e^{0}=1$, and for each element of the group $e^{g}$ there is an inverse $e^{-g}$ such that $e^{g} e^{-g}=1$.

For the DSR1 theory, since the action on momentum space is nonlinear, we do not have a matrix representation of boosts, so this BCH argument is important in arguing that arbitrary rotation and boost transformations form group. However there are some mathematical issues 19. which may require consideration. I shall verify here explicitly the group properties.

\section{GENERAL DSR1 TRANSFORMATIONS}

Equation (3) governs the relation between the energymomentum $\left(\omega^{0}, \vec{k}^{0}\right)$ attributed to a particle by a given inertial observer and the energy-momentum $(\omega, \vec{k})$ attributed to that same particle by another inertial observer. In order to analyze the dependence of $(\omega, \vec{k})$ on the six parameters $\xi_{i}$ that appear in the description of the general rotation/boost transformation with generator $O$ we need to consider the derivatives $\frac{d O}{d \xi_{i}}$ that are done by Feynman formalism

$$
\frac{d}{d \xi_{i}} e^{O}=\int_{0}^{1} e^{(1-s) O} \frac{d O}{d \xi_{i}} e^{s O} d s
$$

Taking into account this rule of derivation ${ }^{3}$ one obtains

$$
\frac{d P_{\mu}}{d \xi_{i}}=\left(\int_{0}^{1} e^{(1-s) O} \frac{d O}{d \xi_{i}} e^{-(1-s) O} d s\right) P_{\mu}+
$$

\footnotetext{
${ }^{3}$ Shortly speaking if we append an index $s$ to two noncommuting operators such that $A_{s} B_{s^{\prime}}$ is equal to $A B$ if $s>s^{\prime}$ and $B A$ if $s<s^{\prime}$ we can equally write $A_{s} B_{s^{\prime}}$ or $B_{s} A_{s^{\prime}}$. The index $s$ should not to be necessarily a discrete one. In particular we can substitute $A$ with $\int_{0}^{1} A_{s} d s$ because for only one operator $s$ is unnecessary and $A \int_{0}^{1} d s=A$, but with the advantage that can be considered like an ordinary function. In our case we can replace $O=$ $\int_{0}^{1} O_{s} d s$. The derivative now is an ordinary derivative of a function, i.e. $\frac{d}{d \xi} e^{\int_{0}^{1} O_{s} d s}=\int_{0}^{1} e^{\int_{0}^{1} O_{s^{\prime}} d s^{\prime}} O_{s} d s$. Sharing out the integration in $d s^{\prime}$ such that $\int_{0}^{1} O_{s^{\prime}} d s^{\prime}=\int_{s}^{1} O_{s^{\prime}} d s^{\prime}+\int_{0}^{s} O_{s^{\prime}} d s^{\prime}$
}

$$
-P_{\mu}\left(\int_{0}^{1} e^{s O} \frac{d O}{d \xi_{i}} e^{-s O} d s\right)
$$

The integrals in $d s$ can be performed by means of Sophus Lie's expansion

$$
\begin{aligned}
e^{t A} B e^{-t A} & =\sum_{0}^{\infty} \frac{t^{n}}{n !} C_{n}(A, B) \\
C_{n+1}(A, B) & =\left[A, C_{n}(A, B)\right] \quad C_{0}(A, B)=B
\end{aligned}
$$

obtaining

$$
\begin{aligned}
\frac{d P_{\mu}}{d \xi_{i}} & =\sum_{n=0}^{\infty} \frac{1}{(n+1) !}\left[C_{n}\left(O, \frac{d O}{d \xi_{i}}\right), P_{\mu}\right]= \\
& =\left[\frac{d O}{d \xi_{i}}, P_{\mu}\right]+\frac{1}{2}\left[\left[O, \frac{d O}{d \xi_{i}}\right], P_{\mu}\right]+\ldots
\end{aligned}
$$

Making use of the algebra commutators, one can rewrite the right-hand side in the form

$$
\frac{d P_{\mu}}{d \xi_{i}}=-i \sum_{j=1}^{6}\left[\beta(\{\xi\})_{i}^{j} O_{j}, P_{\mu}\right]
$$

These formulas are the key to the full description of general rotation and boost transformations in DSR1. As shown in the following in some explicit examples, the coefficients $\beta(\{\xi\})_{i}^{j}$ can be found by recursion formulas.

\section{DETAILED ANALYSIS OF SOME EXAMPLES OF DSR1 TRANSFORMATIONS}

In this section I illustrate the application of the formalism introduced in the preceding section, and, in particular, I verify explicitly the key requirements for group structure of DSR1 transformations.

\section{A. Composition of two Lorentz transformations along the same direction}

Rotations are undeformed in DSR1, so they pose no difficulty. The simplest nontrivial case is the composition of two boosts along the same direction. Let me denote that direction with " 1 ", so that the relevant boost generator is $N_{1}$, and consider a boost with rapidity $\xi_{1}$ and another boost with rapidity $\xi_{2}$. The two transformations in succession yield $P_{\mu}=e^{-i \xi_{1} N_{1}} e^{-i \xi_{2} N_{1}} P_{\mu}^{0} e^{i \xi_{1} N_{1}} e^{i \xi_{2} N_{1}}=$

$$
\begin{aligned}
& \text { one gets } \frac{d}{d \xi} e^{O}=\int_{0}^{1} e^{\int_{s}^{1} O_{s^{\prime}} d s^{\prime}}\left(\frac{d O}{d \xi}\right)_{s} e^{\int_{0}^{s} O_{s^{\prime}} d s^{\prime}} d s= \\
& \int_{0}^{1} e^{(1-s) O} \frac{d O}{d \xi} e^{s O} d s \text { where I eliminated the indexes because the } \\
& \text { operators were all in the right order. For a more exhaustive trac- } \\
& \text { tation see the original paper } 20 \text {. }
\end{aligned}
$$


$e^{-i\left(\xi_{1}+\xi_{2}\right) N_{1}} P_{\mu}^{0} e^{i\left(\xi_{1}+\xi_{2}\right) N_{1}}$ that is again a boost along direction " 1 " with rapidity $\xi_{1}+\xi_{2}$. In this case the application of $(8)$ is very easy because only the first commutator does not vanish. In this way we get the eight differential equations

$$
\frac{d P_{\mu}}{d \xi_{1}}=-i\left(N_{1} P_{\mu}\right) ; \quad \frac{d P_{\mu}}{d \xi_{2}}=-i\left(N_{2} P_{\mu}\right)
$$

which can be analyzed with the same techniques introduced in Ref. [3]. In this paper I use a technique which can be used in alternative to the one of [3] and in most cases give a better chance of finding explicit solutions. The natural way to illustrate this technique is in the simple context considered in this subsection ${ }^{4}$.

Suppose that we know how to construct the transformations done by two boosts in succession. If they are equal to the unique transformation whose differential equations are done by $(10)$ then they must satisfy $(10)$. In this case the two boosts in succession must be equivalent to the single boost transformation with rapidity $\xi_{1}+\xi_{2}$. The end result of two transformations in succession is easily analyzed knowing the action of a single boost 3]. Consider a boost transformation in direction " 1 " $P_{\mu}^{\prime}=P_{\mu}^{\prime}\left(\xi_{2} ; P_{\mu}^{0}\right)$ followed by a second boost transformation in the same direction $P_{\mu}=P_{\mu}\left(\xi_{1} ; P_{\mu}^{\prime}\right)$ we then clearly have

$$
P_{\mu}=P_{\mu}\left(\xi_{1}, \xi_{2} ; P_{\mu}^{0}\right)=P_{\mu}\left(P_{\mu}^{\prime}\left(\xi_{2} ; P_{\mu}^{0}\right), \xi_{1}\right)=P_{\mu}\left(\xi_{1}+\xi_{2} ; P_{\mu}^{0}\right)
$$

Substituting them with the (10) (with the action of generators specified by (11)) one gets an identity. This illustrates the strategy of application of formula (8).

\section{B. Composition of two boost transformations along different directions}

Now I consider a somewhat more complicated case: composition of two boosts along different direction. Let me denote with " 2 " the direction of one of the boosts, with rapidity $\xi_{2}$, and with " 1 " the direction of the other boost, with rapidity $\xi_{1}$. This case involves the generators $N_{1}, N_{2}$ that do not commute and all terms in (8) do not vanish. In particular to express the composition of these two boosts in terms of a single Lorentz transformation it is necessary to calculate the coefficients $a, b, c$ of the BCH $P_{\mu}=e^{-i \xi_{1} N_{1}} e^{-i \xi_{2} N_{2}} P_{\mu}^{0} e^{i \xi_{2} N_{2}} e^{i \xi_{1} N_{1}}=$ $e^{-i\left(a N_{1}+b N_{2}+c M_{3}\right)} P_{\mu}^{0} e^{i\left(a N_{1}+b N_{2}+c M_{3}\right)}$ where $M_{3}$ is proportional to $\left[N_{1}, N_{2}\right]$, i.e. $M_{3}$ is the generator of rotations around direction " 3 ". From the knowledge

\footnotetext{
4 Of course, in the simple case of the composition of two boosts along the same direction, here considered only for illustrative purposes, this technique is not necessary (one could use the technique introduced in [3]).
}

of $a, b, c$ the $\beta_{i}^{j}$ coefficients in (9) can be determined by recursion. In fact, calculating the commutators in (8) one only encounters the operators $O_{1}=$ $N_{1}, O_{2}=N_{2}, O_{3}=M_{3}$ and (8) can be written as $\frac{d P_{\mu}}{d \xi_{i}}=-i \sum_{n=0}^{\infty}\left(\left(\beta_{i}^{1}\right)^{n} N_{1}+\left(\beta_{i}^{2}\right)^{n} N_{2}+\left(\beta_{i}^{3}\right)^{n} M_{3}\right)$. The coefficients at each order $n$ are given by

$$
\begin{aligned}
\left(\beta_{i}^{1}\right)^{n} & =\frac{1}{(n+1) !}\left[c\left(\beta_{i}^{2}\right)^{n-1}-b\left(\beta_{i}^{3}\right)^{n-1}\right] \\
\left(\beta_{i}^{2}\right)^{n} & =\frac{1}{(n+1) !}\left[a\left(\beta_{i}^{3}\right)^{n-1}-c\left(\beta_{i}^{1}\right)^{n-1}\right] \\
\left(\beta_{i}^{3}\right)^{n} & =\frac{1}{(n+1) !}\left[a\left(\beta_{i}^{2}\right)^{n-1}-b\left(\beta_{i}^{1}\right)^{n-1}\right]
\end{aligned}
$$

where $\left(\beta_{i}^{1}\right)^{0}=\frac{d a}{d \xi_{i}},\left(\beta_{i}^{2}\right)^{0}=\frac{d b}{d \xi_{i}},\left(\beta_{i}^{3}\right)^{0}=\frac{d c}{d \xi_{i}}$. Exploiting the fact that the Lorentz algebra is unmodified in DSR the coefficients $a, b, c$ can be determined using matrix representation of the algebra generators (the DSR deformation will then be manifest in the fact that those same coefficients enter different formulas, which take into account the nonlinear realization of the Lorentz algebra ${ }^{5}$ ). Let me start with the matrix representation of the Lorentz generators. They can be constructed expanding the corresponding exponentials. For example consider a boost in direction " 1 "

$$
\begin{aligned}
& P_{\mu}=\left[\exp \left(-i \xi_{1} N_{1}\right)\right]_{\mu \nu}\left(P^{0}\right)^{\nu}=\left[\sum_{n=0}^{\infty} \frac{\left(-i \xi_{1} N_{1}\right)^{n}}{n !}\right]_{\mu \nu} P_{0}^{\nu}= \\
& =\left(\begin{array}{cccc}
\cosh \xi_{1} & \sinh \xi_{1} & 0 & 0 \\
\sinh \xi_{1} & \cosh \xi_{1} & 0 & 0 \\
0 & 0 & 1 & 0 \\
0 & 0 & 0 & 1
\end{array}\right)\left(\begin{array}{c}
\omega^{0} \\
k_{1}^{0} \\
k_{2}^{0} \\
k_{3}^{0}
\end{array}\right)=\exp \left[-i \xi_{1}\left(\begin{array}{cccc}
0 & i & 0 & 0 \\
i & 0 & 0 & 0 \\
0 & 0 & 0 & 0 \\
0 & 0 & 0 & 0
\end{array}\right)\right]\left(\begin{array}{c}
\omega^{0} \\
k_{1}^{0} \\
k_{2}^{0} \\
k_{3}^{0}
\end{array}\right)
\end{aligned}
$$

where the right side has been obtained solving the differential equations that are associated to this boost transformation. In the same manner one gets the matrix form of the other generators here of interest $N_{2}, M_{3}$.

The next step is to get the unique transformation $e^{-i\left(a N_{1}+b N_{2}+c M_{3}\right)}$ yielded by $e^{-i \xi_{1} N_{1}} e^{-i \xi_{2} N_{2}}$. I have to determine the three coefficients $a, b, c$ as functions of $\xi_{1}, \xi_{2}$. To perform this task I can first calculate these transformation in matrix form, the first in terms of $a, b, c$, the second in terms of $\xi_{1}, \xi_{2}$, and then comparing three matrix elements. Following this line of analysis I get a non-linear system of three coupled equations

$$
\begin{aligned}
& \frac{\left(a^{2}+b^{2}\right) \cosh d-c^{2}}{d^{2}}=\cosh \xi_{1} \cosh \xi_{2} \\
& \frac{\left(a^{2}-c^{2}\right) \cosh d+b^{2}}{d^{2}}=\cosh \xi_{1}
\end{aligned}
$$

5 This observation is in agreement with similar results obtained in 17 and will be further confirmed by equs. (16). 


$$
\frac{\left(b^{2}-c^{2}\right) \cosh d+a^{2}}{d^{2}}=\cosh \xi_{2}
$$

where $d^{2}=a^{2}+b^{2}-c^{2}$. A solution of (14) is given by

$$
\begin{aligned}
a & =\sqrt{\cosh \xi_{1}-1} \sqrt{\cosh \xi_{2}+1} \alpha\left(\xi_{1}, \xi_{2}\right) \\
b & =\sqrt{\cosh \xi_{2}-1} \sqrt{\cosh \xi_{1}+1} \alpha\left(\xi_{1}, \xi_{2}\right) \\
c & =\sqrt{\cosh \xi_{1}-1} \sqrt{\cosh \xi_{2}+1} \alpha\left(\xi_{1}, \xi_{2}\right) \\
\alpha\left(\xi_{1}, \xi_{2}\right) & =\frac{\operatorname{arccosh}\left[\frac{1}{2}\left(\cosh \xi_{1} \cosh \xi_{2}+\cosh \xi_{2}-1\right)\right]}{\sqrt{\cosh \xi_{1} \cosh \xi_{2}+\cosh \xi_{2}+\cosh \xi_{1}-3}}
\end{aligned}
$$

One can see that $a\left(\xi_{1}=0, \xi_{2}\right)=0, a\left(\xi_{1}, \xi_{2}=0\right)=\xi_{1}$ and analogous relations hold for $b, c$ as expected. Exploiting equation (8) and recursion formulas (12) one finds in this case

$$
\frac{d P_{\mu}}{d \xi_{1}}=-i\left(N_{1} P_{\mu}\right) ; \frac{d P_{\mu}}{d \xi_{2}}=-i\left(\cosh \xi_{1} N_{2}+\sinh \xi_{1} M_{3}\right) P_{\mu}
$$

Note that these equations are the same that one could find in a purely classical framework. To show that deformed Lorentz transformations satisfy (16) one must construct (following the same technique that we used for the previous example) the complete transformations yielded by two boosts in succession

$$
P_{\mu}=P_{\mu}\left(\xi_{1}, \xi_{2} ; P_{\mu}^{0}\right)=P_{\mu}\left(P_{\mu}^{\prime}\left(\xi_{2} ; P_{\mu}^{0}\right), \xi_{1}\right)
$$

from Ref. [3. It is easy to show that putting (17) in $(16)$ we get again an identity. As in the previous example this shows explicitly one of the group properties of deformed Lorentz transformations.

Exploiting the same technique for the composition of two arbitrary deformed Lorentz transformations one can verify all group properties.

\section{On arbitrary boost transformations}

As a third application of the formula (8) I now consider an arbitrary Lorentz transformation given by exponentiation of the operator $O=-i\left(\xi_{1} N_{1}+\xi_{2} N_{2}\right)$. In this case equation (8) reads

$$
\frac{d P_{\mu}}{d \xi_{1}}=-i\left(A\left(\xi_{1}, \xi_{2}\right) N_{1}-B\left(\xi_{1}, \xi_{2}\right) N_{2}-C\left(\xi_{1}, \xi_{2}\right) M_{3}\right) P_{\mu}
$$

where

$$
\begin{aligned}
& A=1+\frac{\xi_{2}^{2}}{3 !}+\frac{\xi_{1}^{2} \xi_{2}^{2}}{5 !}+\frac{\xi_{2}^{4}}{5 !}+\frac{\xi_{1}^{4} \xi_{2}^{2}}{7 !}+2 \frac{\xi_{2}^{4} \xi_{1}^{2}}{7 !}+\frac{\xi_{2}^{6}}{7 !}+\ldots \\
& B=\frac{\xi_{1} \xi_{2}}{3 !}+\frac{\xi_{1}^{3} \xi_{2}}{5 !}+\frac{\xi_{1} \xi_{2}^{3}}{5 !}+\frac{\xi_{1}^{5} \xi_{2}}{7 !}+2 \frac{\xi_{2}^{3} \xi_{1}^{3}}{7 !}+\frac{\xi_{1} \xi_{2}^{5}}{7 !}+\ldots \\
& C=\frac{\xi_{2}}{2 !}+\frac{\xi_{1}^{2} \xi_{2}}{4 !}+\frac{\xi_{2}^{3}}{4 !}+\frac{\xi_{1}^{4} \xi_{2}}{6 !}+2 \frac{\xi_{1}^{2} \xi_{2}^{3}}{6 !}+\frac{\xi_{2}^{5}}{6 !}+\ldots
\end{aligned}
$$

In this case it is more difficult to derive the exact form of $A, B, C$ from the terms known order by order. However, it is clear that the series converges because the following relations hold

$$
\begin{aligned}
A \leq \cosh \xi_{1} \cosh \xi_{2} & ; \quad|B| \leq\left|\sinh \xi_{1} \sinh \xi_{2}\right| \\
|C| & \leq\left|\sinh \xi_{2} \cosh \xi_{1}\right|
\end{aligned}
$$

for each value of $\xi_{1}, \xi_{2}$. In the same manner we can analyze $\frac{d P_{\mu}}{d \xi_{2}}$ with analogous results.

In this subsection, my analysis gave another illustration of the usefulness of (8) as a useful tool for calculations. In more complicated cases, such as the one considered in this subsection, the formula (\$) is still useful, although close-form all-order results are troublesome. I want to point out that an all-order result on arbitrary boost transformations has been obtained, following an alternative approach (not differential but algebraic), in Ref. [7].

\section{CLOSING REMARKS}

The analysis reported here completes a natural set of consistency checks for the structure of DSR1 in the energy-momentum one-particle sector. DSR1 transformations clearly form a genuine group of symmetries of a plausible energy-momentum space, which is in full agreement with our presently-available low-energy data but predicts observably large new effects for forthcoming experiments [1, 10].

This robust starting point should provide additional motivation for the study of the two-particle sector, where some issues still require further study 1, 5, \&, and for a corresponding spacetime realization [1, 6] of DSR symmetries.

Notice that the same BCH argument developed in section II can be applied in general to all DSR theories presented, and in particular to Magueijo-Smolin DSR2 [4. This suggests that the techniques here proposed for DSR1 might be easily adapted to DSR2 and possible other DSR proposals.

\section{Acknowledgments}

I am grateful to Giovanni Amelino-Camelia for suggesting this research project and for numerous discussions on the development of my studies as part of his supervision of my Laurea thesis. 
[1] G. Amelino-Camelia. Int. J. Mod. Phys. D 11 (2002) 35 arXiv:gr-qc/0012051; Phys. Lett. B 510 (2001) 255 arXiv:hep-th/0012238.

[2] G. Amelino-Camelia, gr-qc/0106004.

[3] N. R. Bruno, G. Amelino-Camelia and J. KowalskiGlikman, "Deformed boost transformations that saturate at the Planck scale," Phys. Lett. B 522 (2001) 133 arXiv:hep-th/0107039.

[4] J. Magueijo and L. Smolin, Phys. Rev. Lett. 88 (2002) 190403. arXiv:hep-th/0112090.

[5] G. Amelino-Camelia, D. Benedetti and F. D'Andrea, hep-th/0201245.

[6] J. Kowalski-Glikman and S. Nowak, hep-th/0203040.

[7] J. Lukierski and A. Nowicki, hep-th 0203065.

[8] S. Judes and M. Visser, gr-qc/0205067.

[9] G. Amelino-Camelia, J. Ellis, N.E. Mavromatos, D.V. Nanopoulos and S. Sarkar, Nature 393, 763 (1998). arXiv:astro-ph/9712103.

[10] J.P. Norris, J.T. Bonnell, G.F. Marani, J.D. Scargle, astro-ph/9912136; A. de Angelis, astro-ph/0009271.

[11] M. Takeda et al., Phys. Rev. Lett. 81, 1163 (1998).
[12] T. Kifune, Astrophys. J. Lett. 518, L21 (1999).

[13] G. Amelino-Camelia and T. Piran, Phys. Rev. D64 (2001) 036005. arXiv:astro-ph/0008107.

[14] J. Lukierski, A. Nowicki, H. Ruegg, and V.N. Tolstoy, Phys. Lett. B264 (1991) 331.

[15] S. Majid and H. Ruegg, "Bicrossproduct structure of kappa Poincare group and noncommutative geometry," Phys. Lett. B 334 (1994) 348 arXiv:hep-th/9405107.

[16] J. Lukierski, H. Ruegg and W. J. Zakrzewski, Annals Phys. 243 (1995) 90 arXiv:hep-th/9312153.

[17] J. Lukierski, H. Ruegg and W. Ruhl, Phys. Lett. B 313 (1993) 357.

[18] I. Batalin, Journ. Math. Phys. 22, 1837 (1981).

[19] Bourbaki, Nicolas, "Lie groups and Lie algebras. Chapters 1-3" Springer-Verlag, Berlin-New York, 1989. 450 pp. ISBN3-540-50218-1 MR89k:17001.

[20] R.P. Feynman, "An Operator Calculus Having Applications in Quantum Electrodynamics", Phys. Review 84 (1951) 108. 